The Astronomical Journal, 115:377-382, 1998 February

(C) 1998. The American Astronomical Society. All rights reserved. Printed in U.S.A.

\title{
KECK SPECTROSCOPY OF THREE GRAVITATIONAL LENS SYSTEMS DISCOVERED IN THE JVAS AND CLASS SURVEYS
}

\author{
Christopher D. FASSNACht AND Judith G. COHEN \\ Palomar Observatory, California Institute of Technology, Mail Stop 105-24, Pasadena, CA 91125; cdf@astro.caltech.edu, jlc@astro.caltech.edu \\ Received 1997 August 1; revised 1997 November 5
}

\begin{abstract}
We present spectra of three gravitational lens systems taken with the Low Resolution Imaging Spectrograph on the W. M. Keck telescopes. All of the systems were discovered in the JVAS and CLASS radio surveys, which were designed to find lenses suitable for measuring $H_{0}$. Previous spectra of these systems had low signal-to-noise ratios, and only one of the source redshifts was secure. Our observations provide unambiguous lens and source redshifts for all of the systems, with $\left(z_{l}, z_{s}\right)=(0.4060,1.339)$, $(0.5990,1.535)$, and $(0.4144,1.589)$ for $\mathrm{B} 0712+472, \mathrm{~B} 1030+074$, and $\mathrm{B} 1600+434$, respectively. The observed image splittings in the systems imply that the masses of the lensing galaxies within their Einstein rings are $5.4 \times 10^{10}, 1.2 \times 10^{11}$, and $6.3 \times 10^{10} h^{-1} M_{\odot}$. The resulting $V$-band mass-to-light ratios for $\mathrm{B} 0712+472$ and $\mathrm{B} 1030+074$, measured inside their Einstein ring radii, are $\sim 10 h(M / L)_{\odot, V}$, slightly higher than values observed in nearby elliptical galaxies. For B1600 +434 , the mass-to-light ratio is $48 h$ $(M / L)_{\odot, V}$. This high value can be explained, at least in part, by the prominent dust lane running through the galaxy. Two of the three lens systems show evidence of variability, so monitoring may yield a time delay and thus a measurement of $H_{0}$.

Key words: distance scale - galaxies: distances and redshifts - gravitational lensing quasars: individual $(\mathrm{B} 0712+472, \mathrm{~B} 1030+074, \mathrm{~B} 1600+434)$
\end{abstract}

\section{INTRODUCTION}

It has been known for many years that gravitational lens systems can in principle be used to determine the values of various cosmological parameters. Refsdal (1964) described a method by which variations in intensity of a multiply imaged background source could be used to determine the Hubble constant. A well-constrained model of the lensing potential can be used to predict differences in light-travel time along the multiple paths from source to observer; knowing the redshifts of the background source and the lensing galaxy for each system is crucial to this method. The ratio of the observed to the predicted time delays is directly proportional to $H_{0}$. Time delays have been measured for two lens systems to date: $0957+561$ (Kundić et al. 1995, 1997b; Oscoz et al. 1997) and 1115+080 (Schechter et al. 1997; Barkana 1997). Combining the measured time delays with current models for these systems has yielded values of $H_{0}$ in the range $\approx 60-70 \mathrm{~km} \mathrm{~s}^{-1} \mathrm{Mpc}^{-1}$ for $0957+561$ (Kundić et al. 1997b; Oscoz et al. 1997; Falco et al. 1997) and $\approx 40-55 \mathrm{~km} \mathrm{~s}^{-1} \mathrm{Mpc}^{-1}$ for $1115+080$ (Schechter et al. 1997; Keeton \& Kochanek 1997; Kundić et al. 1997a; Courbin et al. 1997). Improvements in the time delay measurements and additional constraints on the lens models are expected. The value of Refsdal's method will be proved if several lenses can be used to measure $H_{0}$ and produce consistent values.

The Jodrell Bank-VLA Astrometric Survey (JVAS; Patnaik et al. 1992; Browne et al. 1997b) and the Cosmic Lens All-Sky Survey (CLASS; Jackson et al. 1995; Myers et al. 1995; Browne et al. 1997a; Myers et al. 1998) contain observations of $\sim 10,000$ flat-spectrum radio sources. One of the primary goals of these surveys is to find gravitational lens systems that can be used to measure $H_{0}$. To date, 11 new gravitational lenses have been discovered in the JVAS and CLASS surveys, and 16 lens candidates are being investigated further. The lens and source redshifts have been determined for almost all of the newly discovered lens systems. This paper presents spectra taken at the W. M. Keck Observatory of three of the systems with missing redshifts, B0712 +472, B1030+074, and B1600+434. There is evidence of variability in B0712+472 (Jackson et al. 1997b) and B1600+ 434 (Jaunsen \& Hjorth 1997), so at least two of the systems present the possibility of being used to measure $H_{0}$.

We use $H_{0}=100 h \mathrm{~km} \mathrm{~s}^{-1} \mathrm{Mpc}^{-1}$ and assume $q_{0}=0.5$ throughout this paper.

\section{TARGETS}

Below we present information on previous observations of the lens systems that are discussed in this paper.

\section{1. $B 0712+472$}

This lens system consists of four images of the background source in a typical lensing geometry (see, e.g., Blandford \& Narayan 1992). The maximum image separation is 1 ".27. Radio maps made with the VLA and MERLIN do not resolve the four images (Jackson et al. 1997b). Images taken with the Wide Field Planetary Camera 2 (WFPC2) aboard the Hubble Space Telescope show all four images and, in addition, the lensing galaxy (Jackson, Nair, $\&$ Browne 1997a). Photometry derived from the WFPC2 images measure the lens magnitude as $V \sim 20.2$ and $I \sim$ $20.0,{ }^{1}$ measured in an elliptical aperture with major and minor axes of $2^{\prime \prime}$ and 1", respectively (Jackson et al. 1997b). The total source magnitudes, assuming the images are point sources, are $V \sim 23$ and $I \sim 22.5$. Low signal-to-noise ratio

\footnotetext{
${ }^{1}$ In this paper all WFPC2 F555W and F814W magnitudes have been converted to Johnson $V$ and Cousins $I$ magnitudes, respectively, using transformations in Holtzman et al. (1995).
} 
TABLE 1

\begin{tabular}{|c|c|c|c|c|c|c|}
\hline \multicolumn{7}{|c|}{ OBSERVATIONS } \\
\hline Source & Date & Telescope & $\begin{array}{l}t_{\exp } \\
(\mathrm{s})\end{array}$ & $\begin{array}{l}\text { Slit Width } \\
\text { (arcsec) }\end{array}$ & $\begin{array}{l}\text { P.A. } \\
\text { (deg) }\end{array}$ & $\begin{array}{c}\text { Coverage } \\
\text { (Å) }\end{array}$ \\
\hline $\mathrm{B} 0712+472 \ldots \ldots$ & 1997 Feb 06 & Keck II & 3000 & 0.7 & 75 & $3593-8588$ \\
\hline & $1997 \mathrm{Feb} 14$ & Keck II & 3000 & 0.7 & 79 & $4208-9206$ \\
\hline $\mathrm{B} 1030+074 \ldots \ldots$ & $1997 \mathrm{Feb} 14$ & Keck II & 3000 & 1.0 & 322 & 4211-9208 \\
\hline $\mathrm{B} 1600+434 \ldots \ldots$ & 1996 Jun 18 & Keck I & 3000 & 1.0 & -120 & $4606-9358$ \\
\hline
\end{tabular}

optical spectra of the system, taken with the William Herschel Telescope (WHT), show weak broad emission lines (C IV, C III], $\mathrm{Mg}$ II) yielding a source redshift of $z_{s} \sim$ 1.33 , and a "hint of $\mathrm{Ca} \mathrm{H}$ and $\mathrm{K}$ " absorption, which tentatively yields $z_{l} \sim 0.40$ (Jackson et al. 1997b). The redshifts derived from the WHT spectra, especially that of the lens, are not secure. However, both are confirmed to be correct by the observations described in this paper.

\section{2. $B 1030+074$}

The B1030+074 system consists of two flat-spectrum radio components separated by 1 ".56, with a flux ratio of 15 to 1 (Browne et al. 1997a; Xanthopoulos et al. 1998). These components are unresolved at the milliarcsecond resolution of VLBI. The system was imaged with WFPC2, and both images of the background source are seen, as is the lensing galaxy. Component $\mathrm{A}$, the brighter image, has magnitudes of $V \sim 20$ and $I \sim 19$, and component $B$ is $\sim 2.5-3 \mathrm{mag}$ fainter in each band; the lensing galaxy is estimated to have magnitudes $I \sim 20.5$ and $V \sim 22$ (Xanthopoulos et al. 1998). Spectra taken with the COSMIC spectrograph (Kells et al. 1998) on the Hale Telescope show no clear spectral features (R. C. Vermeulen \& D. S. Womble 1996, private communication).

\section{3. $B 1600+434$}

This system is also a double, with separation 1".4 and a flux ratio of 1.30 to 1 at radio wavelengths (Jackson et al. 1995). Jaunsen \& Hjorth (1997) have imaged the system with the Nordic Optical Telescope and find that the lensing galaxy is an edge-on spiral with $B=23.6, V=22.0$, $R=21.1$, and $I=20.3$. The background source images have total magnitudes of $B=21.9, V=21.7, R=21.1$, and $I=20.4$. Imaging with WFPC2 has shown that the lensing galaxy has a prominent dust lane along its major axis (Jackson et al. 1997a). Previous spectroscopy with the WHT detected broad C IV, C III] and $\mathrm{Mg}$ II emission lines from the background source. These lines yield a source redshift of $z_{s}=1.61$, but no redshift for the lens was determined (Jackson et al. 1995). Jaunsen \& Hjorth (1997) used the lensing galaxy colors to obtain a photometric redshift of $z \sim 0.4$, which is confirmed by the observations presented in this paper.

\section{OBSERVATIONS AND DATA REDUCTION}

All three of the systems were observed with the Low Resolution Imaging Spectrograph (LRIS; Oke et al. 1995) in long-slit mode on the W. M. Keck telescopes. The 300 groove $\mathrm{mm}^{-1}$ grating was used, providing a scale of $2.44 \AA$ pixel $^{-1}$. The long slit was aligned with the major axis of the lensing galaxy $(\mathrm{B} 0712+472$ and $\mathrm{B} 1600+434)$ or the axis defined by the two images of the background source (B1030+074). All objects were observed for $3000 \mathrm{~s}$ total integration time; other details of the observations are given in Table 1. The data were reduced using standard IRAF ${ }^{2}$ routines. The bias levels were estimated using the overscan region on the chip. For the observations of B1600+434, the flat-field frame was constructed from dome flats; in all other cases, exposures with the internal flat-field lamp were used. The spectra were extracted using the IRAF implementation of the "optimal extraction" technique described in Horne (1986) and Marsh (1989). For the 1997 February 7 observation of B0712+ 472 and the 1997 February 14 observation of $\mathrm{B} 1030+074$, the emission from the background source and the lensing galaxy were spatially separated on the slit, so two spectra were extracted for each system. Wavelength calibration was performed using skylines (1997 February 7) or arc lamps taken after each science exposure (all other observations). Observations of the Oke spectrophotometric standard stars G191-B2B and HZ 44 (Oke 1990) were used to remove the response function of the chip. For the data taken on 1996 June 18 and 1997 February 14, exposures of BL Lac objects were used to remove atmospheric absorption features. The individual spectra for each object were weighted by their signal-to-noise ratios and combined.

\section{RESULTS}

The final spectra are shown in Figures 1-5. For each lens system, the spectral features (Table 2) can be explained as the sum of a lens at $z_{l}$ and a background source at $z_{s}$, where a single choice of $z_{l}$ and $z_{s}$ (Table 3 ) suffices. Uncertainties in the redshifts were estimated by taking the rms scatter in the redshifts calculated from the individual spectral lines. For B1600 + 434, the source redshift uncertainty was estimated as half the difference of the redshifts calculated from the two broad emission lines. Further discussion of the individual systems follows below.

\section{1. $B 0712+472$}

The B0712 +472 spectra show a lensing galaxy at $z_{l}=$ 0.4060 and a background source at $z_{s}=1.339$. These results confirm Jackson et al.'s (1997b) tentative redshifts. The lensing galaxy has a typical early-type galaxy spectrum (see, e.g., Kennicutt 1992). The moderately strong $4000 \AA$ break, small equivalent width Balmer absorption lines, and lack of $\mathrm{O}$ II emission indicate that little star formation is occuring in this galaxy. The background source shows broad C III] and $\mathrm{Mg}$ II emission lines typical of a quasar spectrum.

\footnotetext{
${ }^{2}$ IRAF is distributed by the National Optical Astronomy Observatories, which are operated by the Association of Universities for Research in Astronomy, Inc., under cooperative agreement with the NSF.
} 


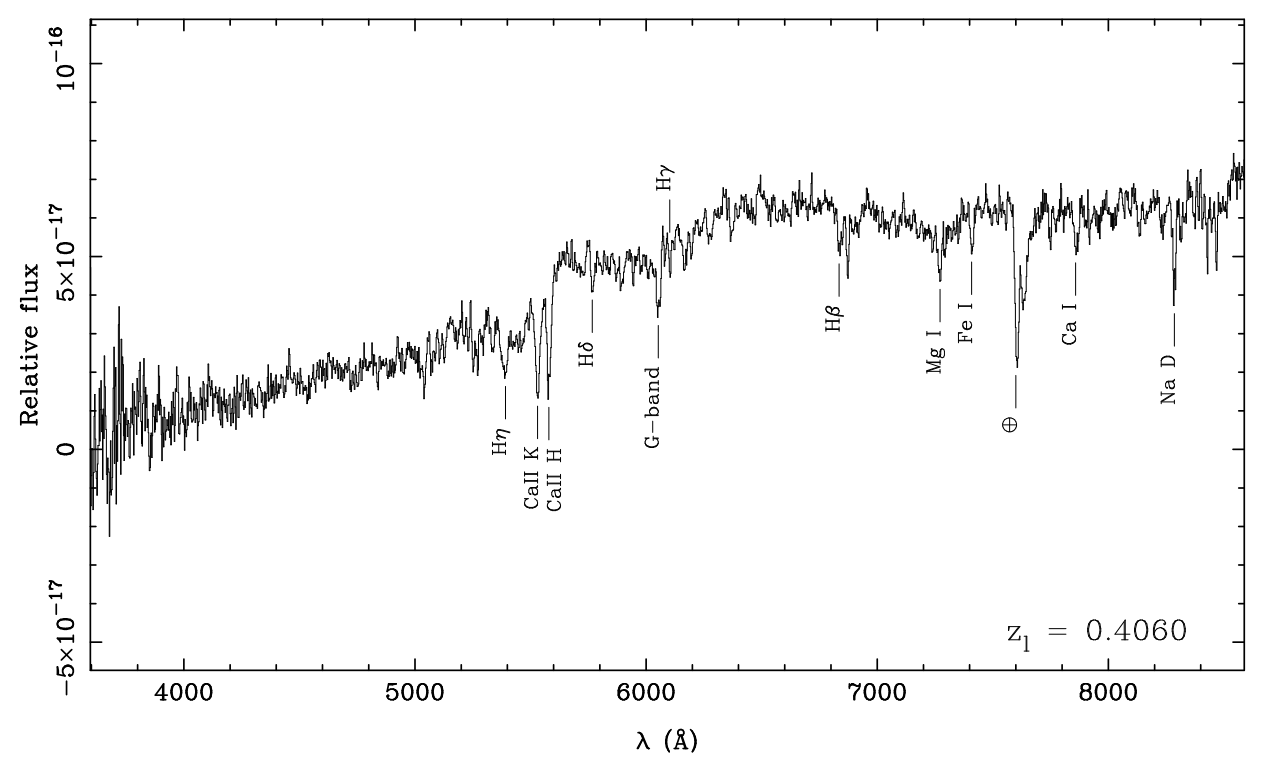

FIG. 1.-Spectrum of lensing galaxy in B0712+472 system, taken on 1997 February 6

\section{2. $B 1030+074$}

The $\mathrm{B} 1030+074$ system contains a lensing object at $z_{l}=$ 0.5990 and a background source at $z_{s}=1.535$. In this system as well, the lensing object spectrum is typical of an early-type galaxy. The background source shows broad $\mathrm{C}$ III] and $\mathrm{Mg}$ II emission lines. The broad emission lines have low equivalent widths, raising the possibility that the background source is a BL Lac-type object. It should be noted, however, that the source spectrum is almost certainly contaminated by some light from the lensing object, which will raise the continuum level and reduce the apparent equivalent widths of the lines.

\section{3. $B 1600+434$}

The $\mathrm{B} 1600+434$ spectrum has the lowest signal-to-noise ratio of the observations presented in this paper. However, both lens and source redshifts $\left(z_{l}=0.4144, z_{s}=1.589\right)$ are clearly determined. The background source is a quasar with broad $\mathrm{C} \mathrm{III]} \mathrm{and} \mathrm{Mg}$ II emission lines, as was seen in Jackson et al. (1995). The lens spectrum is indicative of a later galaxy type than that observed in the B0712+472 and B1030+074 systems. This spectrum has [O II] emission and a less prominent $4000 \AA$ break. This is consistent with images of the lensing galaxy that show that it is an edge-on spiral (Jaunsen \& Hjorth 1997; Jackson et al. 1997a). For later calculations, we will assume that the lensing object is an Sa or $\mathrm{Sb}$ galaxy. However, determining the galaxy type from the spectrum may be complicated by extinction from the dust lane, which runs the length of the observable disk (Jackson et al. 1997a).

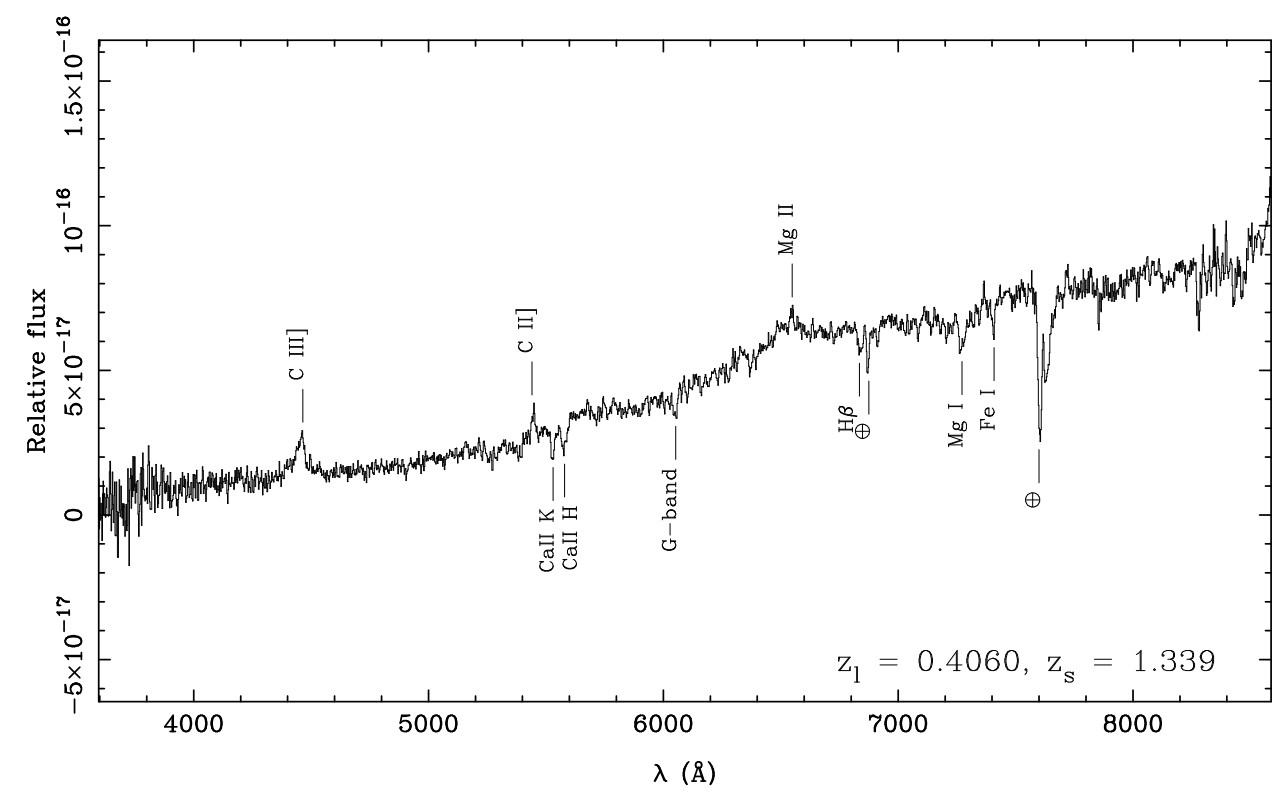

FIG. 2.-Spectrum of background source in B0712 + 472 system, taken on 1997 February 6 . The spectrum also includes a significant amount of light from the extended lensing galaxy. The $\mathrm{C} \mathrm{III],} \mathrm{C}$ II], and $\mathrm{Mg}$ II emission lines are associated with the background source. All other lines are associated with the lensing galaxy. 


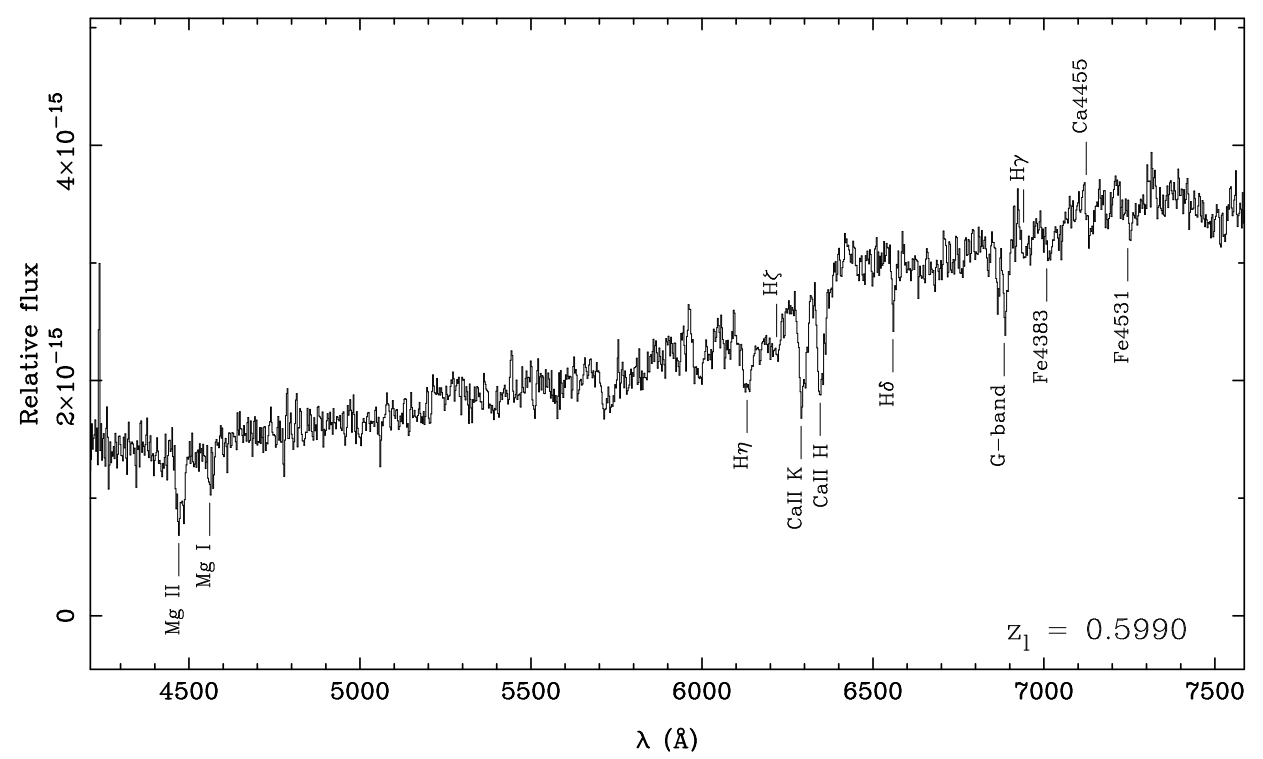

FIG. 3.-Spectrum of lensing galaxy in B1030+074 system, taken on 1997 February 14

\section{DISCUSSION}

In order to use a lens system to measure $H_{0}$, it is necessary to first know the source and lens redshifts. These redshifts are used to derive the angular diameter distances to the source and the lens, which have an inverse dependence on $H_{0}$. The observed image positions and other information can then be used to construct a model of the lensing potential. The lens model is combined with the angular diameter distances to yield predicted time delays:

$$
\Delta t_{i}=\left(1+z_{l}\right) \frac{D_{l} D_{s}}{c D_{1 \mathrm{~s}}}\left[\frac{1}{2}\left|\theta_{i}-\beta\right|^{2}-\phi(\theta)\right]
$$

where $D_{l}, D_{s}$, and $D_{1 \mathrm{~s}}$ are the angular diameter distances to the lens, to the source, and between the lens and source, respectively; $\theta_{i}$ is the image position; $\beta$ is the source position; and $\phi$ is the scaled lensing potential (see, e.g., Hogg \& Blandford 1994). The predicted time delay is proportional to $h^{-1}$ from the ratio of angular diameter distances. Thus, if the background source is variable and time delays can be measured, the ratio between the observed and predicted delays will provide $h$.

We can also use these lenses to study the properties of galaxies at moderate redshifts. For example, the extent of the image splitting by the lens provides a direct estimate of the mass inside the Einstein ring of the lens. For the lens systems discussed in this paper, the Einstein ring radii correspond to physical sizes of $2-3 h^{-1} \mathrm{kpc}$. The mass is estimated as:

$$
M_{\mathrm{E}} \sim 1 \times 10^{12}\left(\frac{D}{1 \mathrm{Gpc}}\right)\left(\frac{\theta_{\mathrm{E}}}{3^{\prime \prime}}\right)^{2} M_{\odot}, \quad D \equiv \frac{D_{l} D_{s}}{D_{1 \mathrm{~s}}}
$$

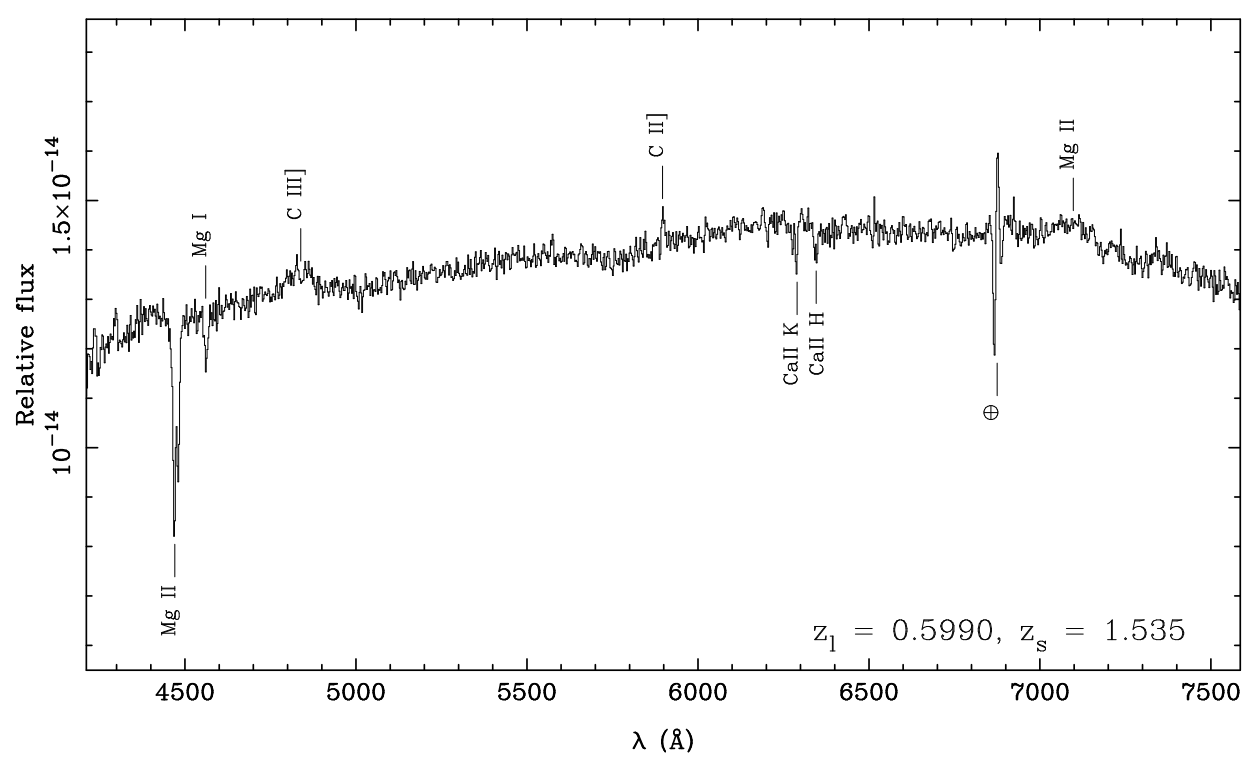

FIG. 4.-Spectrum of background source in B1030+ 074 system, taken on 1997 February 14. The C III], C II], and Mg II emission lines are associated with the background source. All other lines are associated with the lensing galaxy. 


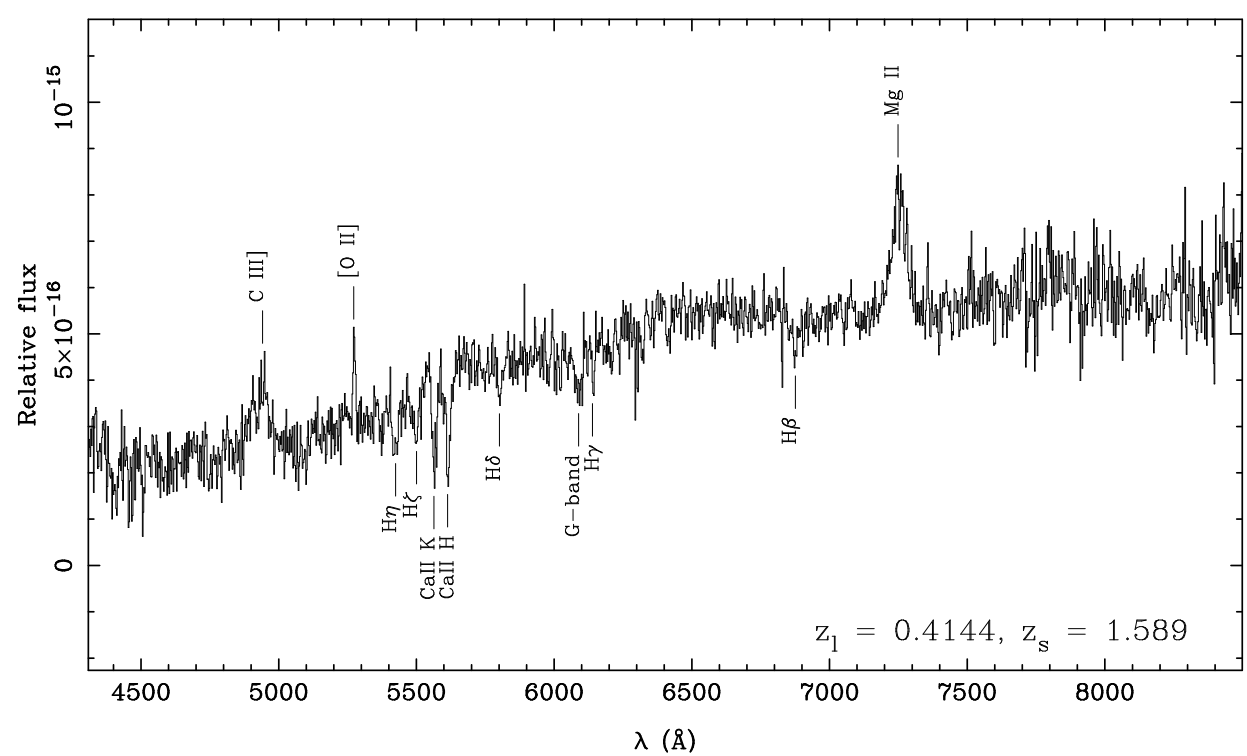

Fig. 5. - Spectrum of B1600+434, containing light from both the lens and the source. The $\mathrm{C}$ III] and $\mathrm{Mg}$ II emission lines are associated with the background source. All other lines are associated with the lensing galaxy.

TABLE 2

DeteCted SPECTRAL Lines

\begin{tabular}{|c|c|c|c|c|c|}
\hline \multirow[b]{2}{*}{ Ion } & \multirow{2}{*}{$\begin{array}{c}\lambda_{0} \\
(\AA)\end{array}$} & \multirow[b]{2}{*}{ Object } & \multicolumn{3}{|c|}{ " OBSERVED WAVELENGTH (Å) } \\
\hline & & & B $0712+472$ & $\mathrm{~B} 1030+074$ & $\mathrm{~B} 1600+434$ \\
\hline $\mathrm{C}$ III] ............ & 1909 & Source & 4461 & 4834 & 4930 \\
\hline $\mathrm{C}$ II] $\ldots \ldots \ldots$ & 2326 & Source & 5448 & 5898 & \\
\hline \multirow[t]{3}{*}{$\mathrm{Mg}$ II .......... } & 2796 & Source & 6540 & 7095 & 7252 \\
\hline & 2796 & Lens & $\ldots$ & 4469 & $\ldots$ \\
\hline & 2802 & Lens & $\ldots$ & 4480 & $\ldots$ \\
\hline $\operatorname{Mg}{ }_{1} \ldots \ldots \ldots$ & 2852 & Lens & $\ldots$ & 4563 & $\ldots$ \\
\hline $\mathrm{O}$ II $\ldots \ldots \ldots \ldots$ & 3727 & Lens & & 5963 & 5274 \\
\hline $\mathrm{H} \eta \ldots \ldots \ldots \ldots$ & 3835 & Lens & 5391 & 6134 & 5418 \\
\hline $\mathrm{H} \dot{\zeta} \ldots \ldots \ldots \ldots$ & 3889 & Lens & & 6212 & 5498 \\
\hline $\mathrm{Ca}$ II K & 3934 & Lens & 5529 & 6288 & 5566 \\
\hline $\mathrm{Ca}$ II $\mathrm{H}^{\mathrm{a}}$. & 3968 & Lens & 5579 & 6345 & 5615 \\
\hline $\mathrm{H} \delta \ldots \ldots \ldots \ldots$ & 4102 & Lens & 5768 & 6560 & 5802 \\
\hline $\mathrm{G}$ band $\ldots \ldots$ & 4300 & Lens & 6053 & 6887 & 6094 \\
\hline $\mathrm{H} \gamma \ldots \ldots \ldots . . .$. & 4340 & Lens & 6104 & 6943 & 6143 \\
\hline $\mathrm{H} \beta \ldots \ldots \ldots \ldots$ & 4861 & Lens & 6839 & $\ldots$ & 6876 \\
\hline \multirow[t]{2}{*}{$\mathrm{Mg} \mathrm{I} \ldots \ldots \ldots . .}$. & 5172 & Lens & 7266 & $\ldots$ & $\ldots$ \\
\hline & 5183 & Lens & 7287 & $\ldots$ & $\ldots$ \\
\hline $\mathrm{Fe}_{\mathrm{I}} \ldots \ldots \ldots \ldots$ & 5269 & Lens & 7409 & $\ldots$ & $\ldots$ \\
\hline $\mathrm{Ca} \mathrm{I}_{\ldots} \ldots \ldots \ldots$ & 5589 & Lens & 7863 & $\ldots$ & $\ldots$ \\
\hline \multirow[t]{2}{*}{$\mathrm{Na}$ I $\mathrm{D} \ldots \ldots}$. & 5890 & Lens & 8283 & $\ldots$ & $\ldots$ \\
\hline & 5896 & Lens & 8290 & $\ldots$ & $\ldots$ \\
\hline
\end{tabular}

${ }^{\text {a }}$ May be blended with $\mathrm{H} \epsilon$ $\lambda 3970$.

TABLE 3

Lens SySTEM PARAMETERS

\begin{tabular}{|c|c|c|c|c|c|c|c|}
\hline System & $z_{l}$ & $z_{s}$ & $\begin{array}{c}D_{l} \\
\left(h^{-1} \mathrm{Mpc}\right)\end{array}$ & $\begin{array}{c}D_{s} \\
\left(h^{-1} \mathrm{Mpc}\right)\end{array}$ & $\begin{array}{c}D_{1 \mathrm{~s}} \\
\left(h^{-1} \mathrm{Mpc}\right)\end{array}$ & $\begin{array}{c}M_{\mathrm{E}^{\mathrm{a}}} \\
\left(10^{10} h^{-1} M_{\odot}\right)\end{array}$ & $\begin{array}{c}(M / L)_{V}^{\mathrm{b}} \\
\left(h[M / L]_{\odot}\right)\end{array}$ \\
\hline B $0712+472$ & $0.4060 \pm 0.0002$ & $1.339 \pm 0.002$ & $668 \pm 0.16$ & $887 \pm 0.042$ & $486 \pm 0.34$ & $5.4 \pm 0.22$ & $8.6 \pm 0.93$ \\
\hline $\mathrm{B} 1030+074 \ldots \ldots$ & $0.5990 \pm 0.0003$ & $1.535 \pm 0.003$ & $784 \pm 0.13$ & $879 \pm 0.16$ & $385 \pm 0.46$ & $12 \pm 0.050$ & $11 \pm 1.2$ \\
\hline $\mathrm{B} 1600+434 \ldots \ldots$ & $0.4144 \pm 0.0003$ & $1.589 \pm 0.006$ & $675 \pm 0.24$ & $876 \pm 0.36$ & $507 \pm 0.53$ & $6.3 \pm 0.25$ & $48 \pm 5.2$ \\
\hline
\end{tabular}

${ }^{a}$ Uncertainties calculated assuming a $1 \%$ uncertainty in image separation.

${ }^{\mathrm{b}}$ Uncertainties calculated assuming a 0.1 mag uncertainty in photometry. 
(see, e.g., Blandford \& Narayan 1992), where $\theta_{\mathrm{E}}$ is the angular radius of the Einstein ring. For these lenses, we find values of $M_{\mathrm{E}} \sim\left(5 \times 10^{10}\right)-\left(1 \times 10^{11}\right) h^{-1} M_{\odot}$.

With the above masses and the photometry for these systems, we can compute approximate mass-to-light ratios for the lensing galaxies. For B0712+472 and B1030+074, the lens magnitudes discussed in $\S 2$ were taken within apertures roughly the size of the Einstein rings in the systems, so we use those values in the following calculations. For B1600+ 434 we have used the WFPC2 images to estimate the lens magnitude in the appropriate aperture, finding $I \sim 21.8$. We converted the observed $I$-band magnitudes to rest frame $V$-band magnitudes using $k$-corrections for the lens redshifts and rest frame $V-I$ colors for typical E/S0 (B0712+472 and B1030+074) and Sa (B1600+434) galaxies. The resulting mass-to-light ratios for $\mathrm{B} 0712+472$ and B1030+ 074 are $\sim 10 h(M / L)_{\odot, V}$. For B1600+434, we find $(M / L)_{E}=48 h(M / L)_{\odot, V}$, confirming the value found by Jaunsen \& Hjorth (1997).

The values of mass-to-light ratio for $\mathrm{B} 0712+472$ and B1030 + 074 are slightly higher than mass-to-light ratios of nearby elliptical galaxies within the same physical radii (see, e.g., van der Marel 1991; Gerhard et al. 1997). We note that we are biased toward finding high-mass systems when looking for gravitational lenses, since these systems have a larger cross section for lensing. Also, the slightly different apertures used to compute the masses and luminosities may be biasing the results toward higher mass-to-light ratios. In addition, the presence of dust in the lensing galaxies would cause some of the light to be lost, thereby increasing the observed mass-to-light ratio. The dust lane in B1600+434 is clearly seen and is responsible, at least in part, for the very high mass-to-light ratio calculated for this system. There is also evidence for absorption by dust in B0712+472 (Jackson et al. 1997a). Evidence for dust has been seen in other lens systems as well (Larkin et al. 1994; Malhotra, Rhoads, \& Turner 1997), indicating that dusty lenses may be quite common. Infrared imaging of these systems would provide more accurate measurements of the mass-to-light ratios.

\section{PROSPECTS FOR MEASURING $H_{0}$}

In order to use these systems to measure $H_{0}$, wellconstrained models of the lensing potentials must be constructed. The following observations could provide data that would aid in the modeling: (1) detecting milliarcsecond-scale structure in the radio images, (2) determining the velocity dispersion of the lensing galaxy (or, in the case of $\mathrm{B} 1600+434$, measuring the galaxy rotation curve), and (3) imaging the systems at high resolution with the Near-Infrared Camera and Multiobject Spectrograph (NICMOS) to provide accurate positions of the lensed images with respect to the lensing galaxy, unbiased by dust extinction.

The final piece of the $H_{0}$ puzzle is measuring time delays between the lensed images. Jackson et al. (1997b) find slight variability in the component fluxes in B0712+472, with a $\sim 30 \%$ overall decrease in flux between 1995 and 1996. The background source in B1600+434 appears to be variable as well. Our VLA observations of B1600+434 show that between 1995 and 1996, the flux densities of the two components decreased by $\sim 50 \%$ and the component flux ratio changed from 1.30 to 1.11 . In addition, Jaunsen \& Hjorth (1997) saw optical variations in B1600+434. Thus, at least two of the sources show variability and, if observed regularly, present the possibility of being used to measure $H_{0}$.

We thank Nicole Vogt, Tony Readhead, and the anonymous referee for insightful comments on the manuscript, and are grateful to Lori Lubin, Mike Pahre, and David Hogg for many helpful discussions. We are indebted to Terry Stickel, Wayne Wack, Chuck Sorenson, and the Keck staff for assistance with the observations. The W. M. Keck Observatory was made possible by a generous grant from the W. M. Keck Foundation. This work is supported by the NSF under grant AST 94-20018.
Barkana, R. 1997, ApJ, 489, 21

Blandford, R. D., \& Narayan, R. 1992, ARA\&A, 30, 311

Browne, I. W. A., et al. 1997a, in Observational Cosmology with the New Radio Surveys, ed. M. N. Bremer, N. Jackson, \& I. Pérez-Fournon (Dordrecht: Kluwer), 305

Browne, I. W. A., Patnaik, A. R., Wilkinson, P. N., \& Wrobel, J. M. 1997b, MNRAS, in press

Courbin, F., Magain, P., Keeton, C. R., Kochanek, C. S., Vanderriest, C., Jaunsen, A. O., \& Hjorth, J. 1997, A\&A, 324, L1

Falco, E. E., Shapiro, I. I., Moustakas, L. A., \& Davis, M. 1997, ApJ, 484, 70

Gerhard, O., Jeske, G., Saglia, R. P., \& Bender, R. 1997, MNRAS, in press

Hogg, D. W., \& Blandford, R. D. 1994, MNRAS, 268, 889

Holtzman, J. A., Burrows, C. J., Casertano, S., Hester, J. J., Trauger, J. T., Watson, A. M., \& Worthey, G. 1995, PASP, 107, 1065

Horne, K. 1986, PASP, 98, 609

Jackson, N., et al. 1995, MNRAS, 274, L25

Jackson, N. J., Nair, S., \& Browne, I. W. A. 1997a, in Observational Cosmology with the New Radio Surveys, ed. M. N. Bremer, N. Jackson, \& I. Pérez-Fournon (Dordrecht: Kluwer), 315

Jackson, N. J., et al. 1997b, MNRAS, in press

Jaunsen, A. O., \& Hjorth, J. 1997, A\&A, 317, L39

Keeton, C. R., \& Kochanek, C. S. 1997, ApJ, 487, 48

\section{REFERENCES}

Kells, W. P., Sivaramakrishnan, A., Dressler, A., Epps, H., Hilyard, G., \& Pardillian, G. 1998, in preparation

Kennicutt, R. C., Jr. 1992, ApJS, 79, 255

Kundić, T., Cohen, J. G., Blandford, R. D., \& Lubin, L. M. 1997a, AJ, 114, 507

Kundić, T., et al. 1995, ApJ, 455, L5

. 1997b, ApJ, 482, 75

Larkin, J. E., et al. 1994, ApJ, 420, L9

Malhotra, S., Rhoads, J. E., \& Turner, E. L. 1997, MNRAS, 228, 138

Marsh, T. 1989, PASP, 101, 1032

Myers, S. T., et al. 1995, ApJ, 447, L5

Oke, J. B. 1990, in preparation

Oke, J. B., et al. 1995, PASP, 107, 375

Oscoz, A., Mediavilla, E., Goicoechea, L. J., Serra-Ricart, M., \& Buitrago, J. 1997, ApJ, 479, L89

Patnaik, A. R., Browne, I. W. A., Wilkinson, P. N., \& Wrobel, J. M. 1992, MNRAS, 254, 655

Refsdal, S. 1964, MNRAS, 128, 307

Schechter, P. L., et al. 1997, ApJ, 475, L85

van der Marel, R. P. 1991, MNRAS, 253, 710

Xanthopoulos, E., et al. 1998, MNRAS, submitted 